SHORT REPORT

\title{
Fatal instability following "odontoid sparing" transoral decompression of a periodontoid pseudotumour
}

\author{
R J Edwards, G W Britz, F G Johnston
}

J Neurol Neurosurg Psychiatry 2002;73:756-758

Pseudotumour of the craniovertebral junction is an uncommon cause of high cervical myelopathy in the elderly. The anterior transoral approach is the preferred means of accessing these lesions. An "odontoid sparing" transoral approach, in which there is only minimal bone removal, is thought to preserve stability of the craniovertebral junction, obviating the need for posterior stabilisation. This report is of an 82 year old man who developed fatal atlanto-axial instability following an odontoid sparing transoral resection of a pseudotumour. This complication has not previously been described and its occurrence has important implications for the surgical management of this condition.

A nteriorly placed extradural mass lesions at the craniovertebral junction causing high cervical myelopathy are uncommon. ${ }^{1}$ Neoplasms are the most frequently encountered masses, although non-neoplastic lesions such as rheumatoid pannus, calcium pyrophosphate dihydrate, gout, and hypertrophic non-union of odontoid fracture have also been described. ${ }^{2-5}$ Peri-odontoid pseudotumour is another non-neoplastic mass lesion, usually encountered in elderly patients with degenerative disease of the cervical spine. ${ }^{2}$

The transoral approach is the established means of accessing anteriorly placed midline extradural pathology and may need to be accompanied by some form of posterior fusion to prevent any subsequent craniovertebral instability. ${ }^{67}$ An odontoid sparing transoral approach for the removal of periodontoid pseudotumours has been described, which was not thought to destabilise the craniovertebral junction, thus obviating the need for postoperative immobilisation and subsequent posterior fixation. ${ }^{2}$ We present a case of a periodontoid pseudotumour in which the patient developed fatal atlantoaxial instability following an odontoid sparing transoral resection without posterior fixation. We discuss the biomechanical reasons for this devastating complication together with the implications for the surgical management of this condition.

\section{CASE REPORT}

An 82 year old man was admitted to hospital with a six month history of a severe, progressive, high cervical myelopathy. Respiratory function was subjectively unaffected. There was no history of rheumatoid arthritis or trauma. The patient had a history of hypertension, which was controlled on drug treatment, but was otherwise fit and well, with no previous history of angina, myocardial infarction, or other major medical problems.

On examination he had a spastic quadriparesis, with increased tone and reflexes in all four limbs, positive Hoffmann's signs bilaterally, and extensor plantar responses. There was wasting of the small muscles of both hands. Dorsal column sensation was impaired in all four limbs.
Magnetic resonance imaging (MRI) (fig lA) showed a smooth, midline, ventral, extradural soft tissue mass, the bulk of which extended posterior to the odontoid process causing cord compression and myelomalacia. Plain radiographs confirmed extensive subaxial spondylotic change but flexion and extension films showed no evidence of instability. Routine preoperative investigations including plasma urea and electrolytes, full blood count, and ECG were normal.

A standard odontoid sparing transoral approach to the mass was used (fig 1B). ${ }^{2}$ This created a "window" through which the pseudotumour, occupying the retro-odontoid space, could be seen and removed by a combination of suction and rongeurs until dural pulsation was obtained. The typical amorphous, xanthomatous pseudotumour material could not be clearly defined as separate from the transverse ligament. This is important because in resecting the lesion-which was not exophytic but rather was within the transverse ligamentinadvertent damage to this stabilising structure may have occurred. The wound was then irrigated and closed in a standard fashion. ${ }^{6}$ There were no intraoperative complications and no orthosis was used.

Histopathological examination of the mass showed degenerate ligament and fibrocartilage, with an associated fibrinous exudate, but no inflammatory infiltrate. No calcium pyrophosphate dihydrate or uric acid crystals were seen. The appearances were consistent with a diagnosis of pseudotumour.

Postoperatively the patient reported an immediate improvement in his neurological symptoms. Postoperative flexionextension films of the cervical spine were not performed because at the time the procedure was not regarded as potentially destabilising. ${ }^{2}$ On the second postoperative day he was found collapsed, having suffered a cardiorespiratory arrest. Cardiopulmonary resuscitation restored a cardiac rhythm and blood pressure but there was no respiratory effort. He was ventilated and transferred to the intensive therapy unit. An EEG was consistent with extensive anoxic brain damage. The patient failed to improve and died on the fifth postoperative day.

At a coroner's necropsy examination there was no cord compression in the neutral position and both the odontoid, which remained attached to the body of $\mathrm{C} 2$, and the anterior arch of $\mathrm{Cl}$ remained intact. However, on digital examination of the foramen magnum with the head flexed the odontoid did compromise the spinal cord. This ligamentous atlanto-axial instability had resulted in severe contusion of the spinal cord at this level. The macroscopic appearances of the contusions were typical and further microscopic analysis was therefore not performed. There was no suggestion of any other possible cause of death; in particular, there was no evidence of a pulmonary embolism, deep vein thrombosis, stroke, or myocardial infarction.

\section{DISCUSSION}

Periodontoid pseudotumours were first described as a separate pathological entity in 1991 by Crockard et al. ${ }^{2}$ They 

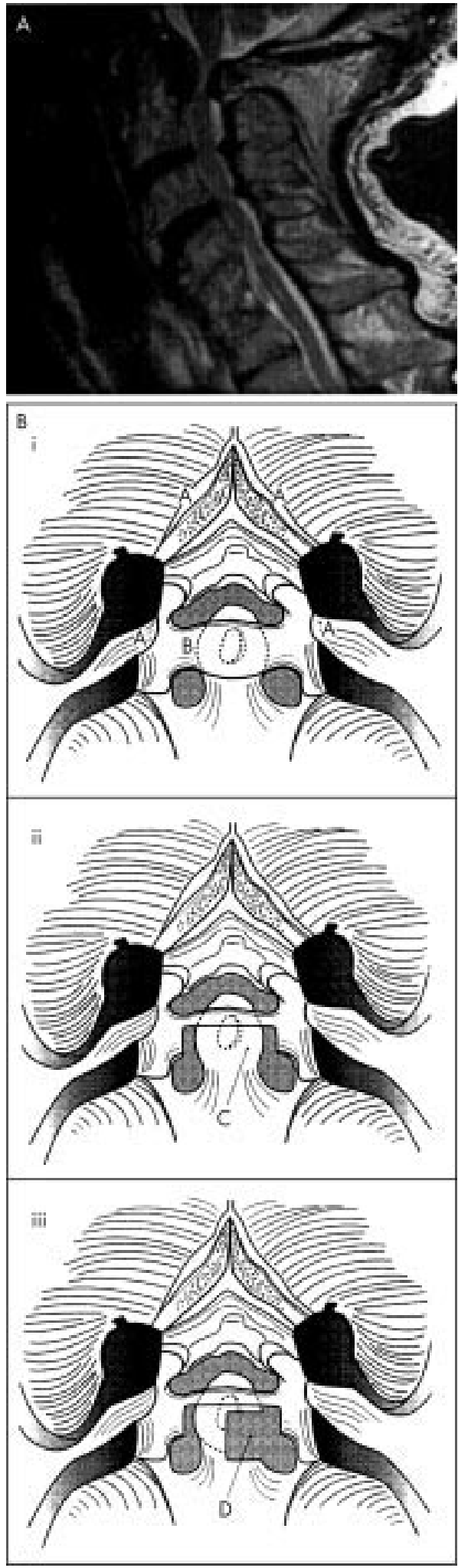

described a mass consisting of amorphous yellowish material, histological examination of which showed degenerate ligament, fibrocartilage, and fibrin but no inflammatory cell infiltrate. It was proposed that these periodontoid pseudotumours arose following a partial tear in the transverse or posterior longitudinal ligaments, with subsequent aberrant attempts at repair resulting in a vicious cycle leading to a progressively enlarging mass. The development of postoperative instability
Figure 1 (A) Sagittal $T_{2}$ weighted magnetic resonance image in the mid-sagittal plane, showing the extradural, posteriorly convex retrodental pseudotumour which is causing marked mid-sagittal compression of the spinal cord. (B) Schematic diagram of the craniovertebral junction showing the extent of bony resection in an odontoid sparing approach to the retro-odontoid space: (i) retracted pharyngeal mucosa (A) exposing the anterior arch of $\mathrm{Cl}(\mathrm{B})$; (ii) the inferior half of the anterior arch of $\mathrm{Cl}$ has been drilled away to expose the underlying odontoid process of $\mathrm{C} 2$ (C); (iii) one half of the odontoid process has been drilled away, preserving the apex with its associated ligamentous attachments, to expose the retro-odontoid space (D)

in our case may reflect this theory that pseudotumour formation occurs secondary to a damaged transverse ligament, which has an important role in maintaining atlantoaxial stability. $^{7-10}$

The ligamentous structures in this region play a vital role in ensuring stability of the craniovertebral junction, preventing subluxation and dislocation, yet preserving functional flexibility. ${ }^{10-13}$ The first line of defence in preventing atlantoaxial dislocation is the transverse ligament, which limits anterior gliding of the atlas in flexion. ${ }^{10}$ Although primarily preventing rotatory instability, the alar ligamentsconnecting the occipital condyles to the dens-provide a second line of defence in preventing impingement of the odontoid process on the cord. ${ }^{11} 1214$ 15 Additional stability is provided to a lesser degree through the tectorial membrane, apical ligament, joint capsules, anterior and posterior atlantooccipital membranes, and the anterior longitudinal ligament. ${ }^{15}$

A standard transoral approach usually involves resection of the anterior arch of the atlas, the odontoid process with its associated apical and alar ligaments, together with the transverse ligament according to the pathology. ${ }^{6}$ Following this type of procedure up to $70 \%$ of patients develop craniovertebral instability ${ }^{16}$ With an odontoid sparing approach the transverse ligament and tectorial membrane may be (partially) resected, although all the other stabilising structures remain intact, obviating the need for postoperative immobilisation and posterior fusion. ${ }^{2}$ Even if the transverse ligament is divided, some biomechanical studies have shown that the alar ligaments, together with the other remaining ligamentous structures and $\mathrm{Cl} / 2$ joint capsules, should provide sufficient stabilisation to prevent cord compromise. ${ }^{8}$ The alar ligaments are relatively strong, and in dynamic tests reported by Dvorak et al ${ }^{11}$ can bear a median axial load of $250 \mathrm{~N}$, approaching that of the transverse ligament, which tolerates a median load of $330 \mathrm{~N}$. Furthermore, these investigators felt that the strength of the alar (but not the transverse) ligaments may have been underestimated. ${ }^{11}$ What is controversial, however, is the degree of excursion permitted by the apical alar ligamentous complex before rupture, following division of the transverse ligament. Fielding et al suggest that the elasticity of the alar ligament may be sufficiently great to cause potential cord compromise. $^{10}$ More recent histological and biomechanical studies, however, have demonstrated the inelastic nature of this ligament and concluded that the alar ligaments can act as a "second line of defence."11 ${ }^{17}$ In reported cases of isolated traumatic rupture of the transverse ligament, instability may develop, ${ }^{10}{ }^{18}$ and some investigators strongly maintain that transverse ligament integrity is essential for craniovertebral stability, although these conclusions are based on observations in conditions in which remaining bone or ligamentous stabilising structures may also be compromised. ${ }^{79}$

This case shows that instability can occur after resection of a pseudotumour using this odontoid sparing approach. The reason may be related to the underlying pathological basis of pseudotumour formation. Fielding et al found considerable age independent variability in the strength of the normal transverse ligament when subjected to flexion loading. ${ }^{10}$ 
Twenty per cent of the ligaments in their series were unaccountably weak, rupturing after a force only $25 \%$ of that required for the remainder. It may be that it is this group of patients with inherently weak ligaments who are predisposed to pseudotumour development, owing to an increased propensity to ligamentous injury following relatively minor trauma. It is also possible that secondary stabilising ligaments are similarly affected and are therefore unable to provide sufficient structural stability following surgical division of the transverse ligament.

An alternative explanation for the development of instability is that a long forgotten whiplash injury may have caused a partial transverse ligament tear, which after many years of attempted healing results in pseudotumour formation. The mechanism of whiplash injury, particularly if occurring with the head in maximum rotation, could also result in stretching or rupture of the alar ligaments, ${ }^{14}{ }^{17}$ which may not then be able to provide adequate secondary stability following pseudotumour removal.

We believe that this case shows that pseudotumours arise from weakened ligaments, and the occurrence of postoperative instability was to some extent an expression of the natural progression of this degenerating process. The surgical implications are that even following odontoid sparing resection, stability in these cases cannot be presumed, although the risk of instability is substantially reduced. Patients should be immobilised in an orthosis postoperatively and undergo early and delayed flexion/extension plain radiographs of the cervical spine, followed by posterior fusion if instability is demonstrated.

\section{Authors' affiliations}

R J Edwards, Department of Neurosurgery, Frenchay Hospital, Bristol, UK

G W Britz, Department of Neurological Surgery, University of Washington School of Medicine and Harborview Medical Center, Seattle, Washington, USA

F G Johnston, Department of Neurosurgery, Atkinson Morley's Hospital, London SW2O, UK

Competing interests: none declared
Correspondence to: Mr F G Johnston, Department of Neurosurgery, Atkinson Morley's Hospital, Copse Hill, London SW20 ONE, UK edwards.r@virgin.net

Received 14 December 2001

In revised form 7 August 2002

Accepted 21 August 2002

\section{REFERENCES}

1 Editorial: Missed foramen-magnum tumours. Lancet 1973; ii: 1482

2 Crockard HA, Sett P, Geddes JF, et al. Damaged ligaments at the craniocervical junction presenting as an extradural tumour: a differential diagnosis in the elderly. J Neurol Neurosurg Psychiatry 1991;54:817-21.

3 Moskovich R, Crockard HA. Myelopathy due to hypertrophic nonunion of the dens: case report. J Trauma 1990;30:222-5.

4 Vinstein AL, Cockerill E. Involvement of the spine in gout. A case report. Radiology 1972;103:311-12.

5 Zunkeler B, Schelper R, Menezes AH. Periodontoid calcium pyrophosphate dihydrate deposition disease: "pseudogout" mass lesions of the craniocervical junction. J Neurosurg 1996;85:803-9.

6 Crockard HA. The transoral approach to the base of the brain and upper cervical cord. Ann R Coll Surg Engl 1985;67:321-5.

7 Dickman CA, Crawford NR. Biomechanics of the craniovertebral junction. In: Dickman CA, Spetzler RF, Sonntag VKH, eds. Surgery of the craniovertebral junction. New York: Thieme, 1998:59-80.

8 Steel $\mathrm{HH}$. Anatomical and mechanical considerations of the atlanto-axial articulations. J Bone Joint Surg Am 1968:50-A:1481-2.

9 Dickman CA, Mamourian A, Sonntag VK, Drayer BP. Magnetic resonance imaging of the transverse atlantal ligament for the evaluation of atlantoaxial instability. J Neurosurg 1991;75:221-7.

10 Fielding JW, Cochran GB, Lawsing JF, et al. Tears of the transverse ligament of the atlas. A clinical and biomechanical study. J Bone Joint Surg Am 1974;56: 1683-91.

11 Dvorak J, Schneider E, Saldinger P, et al. Biomechanics of the craniocervical region: the alar and transverse ligaments. J Orthop Res 1988;6:452-61.

12 Panjabi $M$, Dvorak J, Crisco J, et al. Flexion, extension, and lateral bending of the upper cervical spine in response to alar ligament transections. J Spinal Disord 1991;4:157-67.

13 Panjabi M, Dvorak J, Crisco JJ, et al. Effects of alar ligament transection on upper cervical spine rotation. J Orthop Res 1991;9:584-93.

14 Dvorak J, Panjabi MM. Functional anatomy of the alar ligaments. Spine 1987:12:183-9.

15 White AA, Panjabi MM. The clinical biomechanics of the occipitoatlantoaxial complex. Orthop Clin North Am 1978;9:867-78.

16 Dickman CA, Locantro J, Fessler RG. The influence of transoral odontoid resection on stability of the craniovertebral junction. J Neurosurg 1992:77:525-30.

17 Saldinger P, Dvorak J, Rahn BA, et al. Histology of the alar and transverse ligaments. Spine 1990;15:257-61.

18 Floman Y, Kaplan L, Elidan J, et al. Transverse ligament rupture and atlanto-axial subluxation in children. J Bone Joint Surg $\mathrm{Br}$ 1991;73:640-3. 